\title{
Little justification for the removal of pathology-free third molars
}

\author{
Song F, Landes D P, Glenny A M, Sheldon TA. Prophylactic removal of impacted third molars: an assessment of \\ published reviews. BrDent J 1997; 182: 339-346
}

Objective To critically evaluate reviews about the appropriateness of prophylactic removal of impacted third molars.

Data sources Electronic searches of MEDLINE, Embase, Bids. Hand searches of Index to Dental Literature and references cited in relevant papers.

\begin{abstract}
Study selection Reviews of research literature, addressing pathology and/or symptoms associated with impacted third molars or outcomes of surgical removal published after 1985. Editorials, letters news and comment together with papers where the literature review was not the main element were excluded. Relevance was checked independently by two reviewers. Quality of search method of each review included was assessed as were the inclusion criteria and presentation.
\end{abstract}

Results 12 reviews fitted the entry criteria, 9 covered general issues while 3 focussed on crowding associated with impaction. A further 5 reviews were excluded. Only one review outlined the search method and criteria for inclusion of the studies.

Third molars and crowding: Association not significant enough to warrant removal.

Pericoronitis: No standard definition used, best estimate of prevalence $10 \%$

\begin{abstract}
Cysts and tumours: Range of incidence quoted $0-11 \%$ however difficult to judge reliability of these estimates due to lack of detail in reviews identified.

Caries and periodontal disease: Stated to be common but little objective evidence presented. One review giving an incidence of periodontitis ranging from $1-4.5 \%$. However because of different definitions of periodontitis comparisons difficult.

Complications and risks: Sensory nerve damage 1-6\%; alveolar osteitis (dry socket) 1-3.5\%; Serious post operative infection was estimated as 25 per 100,000 operations.
\end{abstract}

Conclusion In the absence of good evidence to support prophylactic removal there appears to be little justification for the removal of pathology free impacted third molars.

\begin{abstract}
Address F. Song, NHS Centre for Reviews and Dissemination, University of York, UK.
\end{abstract}

Sources of funding: Based on a report commissioned by the Royal College of Surgeons of England. The Department of Health funds the NHS Centre for Reviews and Dissemination, York.

\section{Commentary}

Song and his colleagues at the NHS Centre for Reviews and Dissemination at the University of York have assessed the evidence of effectiveness of prophylactic removal of third molars, with reference to 12 published reviews objectively selected from the substantial literature on third molars. Two reviews of relatively poor quality supported prophylactic removal whereas the great majority concluded that there was a lack of evidence to support this procedure.

There are a number of important issues which this article raises, principal of which is that the treatment intervention being considered is prophylactic and not therapeutic. The purpose is the prevention of future disease and parallels with the scientific basis of screening for risk factors and intervention in high risk, but disease free individuals are therefore more relevant than parallels with randomised trials of therapeutic interventions.

Because there is a need to balance the benefits of prophylactic removal with surgical risks, the authors very appropriately cite published decision analyses which evaluate different outcomes using a common method, which have both concluded that, taking known risks into account, the appropriate treatment strategy is non-intervention.

This critical appraisal of published evidence is of help to clinicians faced with choices about intervention, to commissioners faced with apparently conflicting views about the need for what has been a frequently performed elective procedure and to purchasers of both public and private health care for whom quality and cost effectiveness are of critical importance. There is also help here for teachers who are now armed with the weight of evidence against prophylactic surgery and an easily accessible source of information about the true prevalence of pathology associated with third molars: much lower than has often been taught.

For researchers, the key question is whether prediction of future pericoronitis, periodontal disease, cystic change and unrestorable caries in individual patients can be improved to the extent which is necessary for prophylactic surgery reasonably to be offered to them as the treatment of choice. This review shows that current knowledge does not constitute grounds for removal of disease-free third molars in order to prevent these conditions or crowding.

Jonathan P Shepherd

Professor of Oral and Maxillofacial

Surgery, University of Wales College of

Medicine, Heath Park, Cardiff, UK 